\title{
Actitudes y conocimientos acerca de ética en investigación con animales en estudiantes de Medicina Veterinaria en dos universidades en Lima-Perú
}

\author{
Attitudes and knowledge about ethics in animal research in students of Veterinary Medicine in two universities \\ in Lima-Peru
}

Melanie Caballero García ${ }^{1}$, Juan Espinoza Blanco², Néstor Falcón Pérez ${ }^{3}$

\section{RESUMEN}

El objetivo principal de este estudio fue determinar las actitudes y conocimientos acerca de ética en investigación con animales en estudiantes del primer al quinto año de medicina veterinaria de dos universidades de Lima. La metodología consistió en la elaboración de un instrumento, encuesta, que fue validada por medio de un grupo de expertos y las pruebas estadísticas requeridas como la $\mathrm{V}$ de Aiken, el alfa de Cronbach y análisis factorial, se tuvieron en cuenta todas las consideraciones éticas y fue aprobado por el Comité Institucional de Ética de la Universidad Peruana Cayetano Heredia. No se encontró diferencias significativas en cuanto a conocimientos de ética en investigación con animales, según género y año de estudio; sin embargo, si se encontró diferencias en estas covariables en actitudes. Se encontró un alto nivel de actitudes positivas, por lo que se propone potenciar dichas actitudes impartiendo conocimientos sobre ética en investigación con animales en la formación académica del médico veterinario.

PALABRAS CLAVE: Etica, investigación, bienestar animal, veterinaria.

\section{SUMMARY}

The main ojective was to determine the level of ethic knowledge and attitudes on research with animals in a sample of students from the first to fifth year of Veterinary Medicine from two universities in Lima, Peru. The methodology consisted in the elaboration of an instrument, survey, that was validated with group of experts and tested with the V of Aiken, the Alfa of Cronbach and factorial analysis; and finally approved by the CIE (The Committee of Ethics in Research) of the University. No significant differences were found about knowledge nor between genders, neither between years of study, however were these two covariates different regarding attitudes. A high level of positive attitudes was found. It is proposed to strengthen and enhance knowledge and attitudes about ethics in animal research throughout college formation.

KEY WORDS: research, ethic, welfare animal, veterinary.

\footnotetext{
${ }^{1}$ Laboratorio de Investigación y Desarrollo, Unidad de Negocio Feed - QUIMTIA S.A., Lima, Perú.

${ }^{2}$ Facultad de Medicina Veterinaria, Universidad Nacional Mayor de San Marcos. Lima, Perú.

${ }^{3}$ Facultad de Medicina Veterinaria y Zootecnia, Universidad Peruana Cayetano Heredia. Lima, Perú.
} 


\section{INTRODUCCIÓN}

La ética es la aplicación de la razón a un conjunto de creencias, hábitos y códigos de normas que cada cultura posee por lo que su aplicación modula la actitud que las personas pueden desarrollar ante la circunstancia de proceder éticamente en las investigaciones tanto en humanos como en animales (Cardozo de Martínez, Mrad de Osorio y Martínez, 2007; Serpell, 2005). En el caso de animales, se debe de considerar que estos tienen la capacidad de sentir y sufrir, por ello es necesario estudiar el comportamiento normal del animal para identificar reacciones y cambios frente al dolor para poder aliviarlo (Cardozo et al., 2007; Lolas, Quezada y Rodriguez, 2006; Smith y Jennings, 1998) Los comités de ética en investigación con animales, se encargan de velar por los aspectos éticos y el cumplimiento del protocolo sobre el cuidado y manejo de animales de experimentación en proyectos de investigación y actividades de docencia. La mayoría de países han incorporado este tipo de organización y en otros países como el Reino Unido, Canadá, USA, no solo existen los Comités Institucionales de Ética Animal sino también organizaciones anexas que hacen cumplir las condiciones que aprobó el Comité de Ética (Kimwele, Matheka y Ferdowsian, 2011; Fenwick, Danielson y Griffin, 2011; Leenaars et al., 2009; Lolas et al., 2006).

El Código de Nuremberg, la Declaración de Helsinki, el Reporte de Belmont y las Normas CIOMS, consideran que cualquier experimento realizado en seres humanos debe ser diseñado y basado en resultados de investigación animal. En el caso de la medicina veterinaria existen principios éticos como las Tres Rs a las que se sugiere deban de identificarse los investigadores y docentes del área (Cardozo et al., 2007). Dicho principio consiste en la Reducción, Reemplazo y Refinamiento del uso de animales. Reducción del número de animales usados en proyectos considerando metodología estadística avanzada, mejor selección del modelo animal, banco de datos, etc. Reemplazando si es posible por otro tipo de modelos como sistemas in vitro, animales muertos, técnicas modernas, etc., y Refinando los procedimientos de manipulación mediante el estudio de su comportamiento, considerando cuidados y bienestar animal, capacitación del personal, detectando el dolor, eutanasia anticipada, etc. (Fenwick et al., 2011; Lewis, 2009; Howard, Hudson y Preziosi, 2009; Huertas, 2009).

La ética en investigación con animales, es una disciplina de gran valor, sin embargo sus fundamentos son desconocidos por un número significativo de estudiantes y profesionales de la salud (Paul y Podberscek, 2000; Perry, 2007; Kimwele et al., 2011. El actuar éticamente se debe impartir desde el inicio de la formación del estudiante lo que redundará en una mejora de conocimientos y prácticas en la investigación con animales generándose así el desarrollo de valores que incluye respeto, dignidad, responsabilidad, justicia y equidad; creando una línea ética en el proceder del investigador (Kimwele et al., 2011; Fernández y Heuze, 2007; Self, Baldwin Jr, Wolinsky y Shadduck, 1993; Carlsson, Hagelin, Höglund y Hau, 2001; Main, Thornton y Kerr, 2005; Kitto, Griffiths y Pesek, 2003).

Es necesario brindar mayor información a los estudiantes acerca de los principios de bienestar animal y sus cuidados en los experimentos; esto se debe impartir desde el inicio de su formación lo que redundará en una mejora de conocimientos y prácticas en estos procesos de investigación con animales, generándose así el desarrollo de valores y una ética en el proceder del investigador.

En este contexto, el objetivo del estudio fue determinar los conocimientos y actitudes acerca de la ética en investigación con animales entre estudiantes de Medicina Veterinaria en dos universidades de Lima.

\section{MATERIAL Y MÉTODOS}

El estudio observacional descriptivo de corte transversal se realizó en dos Facultades de Medicina Veterinaria de la ciudad de Lima, de las cuales una fue pública y la otra privada. La población objetivo estuvo compuesta por la totalidad de estudiantes del primer al quinto año de ambas facultades. Al momento del estudio se contaban con aproximadamente 365 en la universidad pública y 249 estudiantes en la universidad privada. La muestra se limitó al total de estudiantes de ambas instituciones que aceptaron participar voluntariamente en el estudio.

El estudio implicó la elaboración de un instrumento de recolección de información (encuesta) que contó con 16 preguntas, de las cuales 10 fueron de conocimientos (opciones múltiples) y seis actitudinales (escala de Likert abreviada). Las preguntas de conocimientos versaron en los siguientes temas: (1) Las " 3 Rs" de Burch y Russell, (2) organismo en el Perú que protege al animal de experimentación, (3) situaciones en las que está prohibido el uso de animales vivos para investigación, (4) función del comité de ética en animales, (5) los términos minimización o terminación 
del sufrimiento del animal, (6) consideración del investigador que trabaja con animales de laboratorio, (7) conocimiento acerca de la existencia de un organismo regulador de investigaciones con animales en su institución, (8) principio ético según la normas CIOMS, (9) cuando se debe de iniciar el estudio con animales, (10) las características que debe tener un investigador. En cuanto a las preguntas actitudinales, sus interpretaciones se presentan en la tabla 1.

El instrumento fue validado por expertos en contenido, estructura y confiabilidad. Estos profesionales fueron especialistas en el tema de ética en investigación con animales o en validación de instrumentos. Para la validación de contenido se elaboró una ficha que contenía preguntas acerca de la redacción y la importancia que tenían los ítems, para responder al objetivo del estudio. Esta fue entregada a cada uno de los especialistas y después del análisis de las respuestas se mejoraron las preguntas que se encontraban observadas.

En relación a la validación del instrumento, se aplicó la prueba estadística $\mathrm{V}$ de Aiken, obteniendo una concordancia de 0,8 que representa una calificación de muy buena. Para la validación de estructura se utilizó 30 encuestas y se realizó el análisis factorial, mediante el cual se determinó su agrupación en ocho factores que respondían al objetivo del cuestionario. No fue necesario discriminar ningún ítem y la confiabilidad obtenida aplicando el Alpha de Cronbach fue de 0.61.

Antes de la ejecución de las encuestas, los estudiantes recibieron una hoja de consentimiento informado, el cual contenía una breve explicación sobre el objetivo del trabajo y los compromisos que los participantes y equipo investigador asumían. Para su aplicación se solicitó los permisos correspondientes a las autoridades de ambas universidades, quienes a su vez programaron fecha y hora de aplicación del instrumento.

La información de las encuestas contestadas fue transferida a una base de datos en el programa Microsoft Excel. Los resultados se analizaron con el programa estadístico SPSS versión 19 mediante estadística descriptiva, resumiendo los resultados mediante frecuencias absolutas y relativas. Para identificar la relación entre la variable principal y las covariables se utilizó la prueba estadística de Chi Cuadrado.

La investigación fue aprobada para su ejecución por el Comité Institucional de Ética en Investigación de la Universidad Peruana Cayetano Heredia.

Tabla 1. Interpretación de las preguntas actitudinales de la encuesta del estudio de investigación.

Pregunta 11: TÓPICO: INICIATIVA DE REDUCCIÓN

o Estar de acuerdo equivale a no considerar la posibilidad de emplear menos animales para obtener el mismo resultado.

o No estar de acuerdo equivale a estar dispuesto a cumplir con una de las 3Rs, la referente a la reducción del tamaño de muestra.

Pregunta 12: TÓPICO: SELECCIÓN POR ESPECIE

o Si cambiaría significa variar su decisión dependiendo de la especie experimental a usar

o No cambiaría, equivale a que el principio es el mismo independiente del tipo de animal con el que trabaje

Pregunta 13: TÓPICO: ASPECTOS ÉTICOS

o El alumno estaría de acuerdo en cumplir con los aspectos éticos o prioriza el objetivo experimental sobre el bienestar del animal.

Pregunta 14: TÓPICO: DINERO

o El alumno decide entre priorizar el bienestar animal o incrementar el costo del experimento.

Pregunta 15: TÓPICO: MATERIAL FRESCO EXPERIMENTAL

o El alumno decide entre utilizar mayor muestra experimental en función de mayor experiencia.

Pregunta 16: TÓPICO: REDUCCIÓN

o Aplicación del método de Reducción. 


\section{RESULTADOS}

La muestra tuvo un total de 317 encuestas, perteneciendo a la universidad privada $178(56,2 \%)$ y a la universidad pública 139 (43,8\%) encuestas. Por aspectos éticos no se comparó resultados entre ambas universidades. La mayoría de los encuestados fueron mujeres (60,6\%); así como la mayoría de las encuestas provinieron de los estudiantes de primer $(26,3 \%)$ y cuarto año $(25,0 \%)$. En la tabla 2 se muestran las características de los participantes en el estudio.
La población encuestada no conoce el significado de las 3 Rs, el término punto final, y además desconoce que organismos regulan los aspectos éticos en investigación. En cuanto a actitudes, los estudiantes de primer año mostraron tener una mayor sensibilidad por el bienestar de los animales que los de quinto año. En el caso de sustituir las truchas por perros, la mayoría de estudiantes del primer y el quinto año no cambiarían su opinión, mostrando que los estudiantes de primeros años poseen una actitud positiva hacia las 3Rs, específicamente a la Reducción. Por otro lado

Tabla 2. Características de los estudiantes de Medicina Veterinaria de dos universidades de Lima, participante en el estudio $(n=317)$

Variable

Centro de estudios

Universidad 1

Universidad 2

Género

Femenino

Masculino

Año de estudio

Primero
Segundo
Tercero
Cuarto
Quinto

$\mathrm{n}$

84

61

45

79

48
$\%$

56,2

43,8

60,6

39,4

26,5

19,2

14,2

24,9

15,1

Tabla 3. Aciertos en conocimientos de ética en investigación con animales en los estudiantes de dos universidades de Lima $(\mathrm{n}=317)$.

\begin{tabular}{|c|c|c|}
\hline \multirow{2}{*}{ Preguntas } & \multicolumn{2}{|c|}{ Respuestas contestadas correctamente } \\
\hline & $\mathrm{n}$ & $\%$ \\
\hline - Conoce las Tres Rs de Burch y Russel. & 49 & 15,5 \\
\hline - Conoce el Organismo regulador de ética en investigación en Perú. & 198 & 62,5 \\
\hline - En qué casos está prohibido el uso animal. & 175 & 55,2 \\
\hline - Cuál es la función del Comité de ética en investigación. & 279 & 88,0 \\
\hline - Conoce el termino Punto Final. & 39 & 12,3 \\
\hline - Cuáles deben ser las consideraciones éticas del investigador. & 218 & 68,8 \\
\hline $\begin{array}{l}\text { - Conoce si su universidad cuenta con Comité de ética en investigación / } \\
\text { ¿Cómo se llama? }\end{array}$ & 42 & 13,2 \\
\hline - Considera un principio ético según las normas CIOMS. & 190 & 59,9 \\
\hline - El investigador debe únicamente iniciar el estudio cuando... (condiciones). & 219 & 69,1 \\
\hline - Qué características debe tener el investigador. & 241 & 76,0 \\
\hline
\end{tabular}


Tabla 4. Actitudes en relación a la ética en investigación con animales de estudiantes de Medicina Veterinaria de dos universidades de Lima $(n=317)$.

\begin{tabular}{llcccccc}
\hline & & \multicolumn{2}{c}{ De acuerdo } & \multicolumn{2}{c}{ En desacuerdo } & \multicolumn{2}{c}{$\begin{array}{c}\text { No sabe / No } \\
\text { responde }\end{array}$} \\
\cline { 3 - 7 } Casos propuestos & $\mathrm{n}$ & $\%$ & $\mathrm{n}$ & $\%$ & $\mathrm{n}$. & $\%$ \\
- & 59 & 18.6 & 232 & 73.2 & 26 & 8.2 \\
- & Caso Trucha arcoíris & 53 & 16.7 & 242 & 76.3 & 22 & 6.9 \\
- & Caso con perros & 261 & 82.3 & 34 & 10.7 & 22 & 6.9 \\
- & Analgesia negada & 39 & 12.3 & 234 & 73.8 & 44 & 13.9 \\
- & Bienestar animal & 53 & 16.7 & 217 & 68.5 & 47 & 14.8 \\
- & Clase practica & 229 & 72.2 & 22 & 6.9 & 66 & 20.8 \\
\hline
\end{tabular}

los estudiantes del quinto año presentan una actitud opuesta, incluso no diferencian ni cambian su actitud considerando el tipo de especie animal.

Respecto al uso de animales en las prácticas, indistintamente del número y tipo animales vivos que se utilizaría, la mayoría de los estudiantes estaban en desacuerdo, sobre todo las mujeres y los estudiantes del primer, segundo y tercer año.

No se encontró diferencia por efecto del género del estudiante sobre los conocimientos sobre ética en investigación con animales (tabla 3). En cuanto a las actitudes, la mayoría de hombres y mujeres están en desacuerdo de utilizar un número mayor de lo necesario de animales de experimentación (tabla 4). Así mismo, la mayoría de estudiantes están en desacuerdo sobre dejar de tomar decisiones sobre el bienestar animal por un factor económico.

\section{DISCUSION}

Las preguntas de conocimientos se elaboraron a partir del contenido del libro de Ética en Investigación publicado con el auspicio de la Organización Panamericana de la Salud (Cardozo et al., 2007) así como fuentes bibliográficas relacionadas al tema. Las preguntas de actitudes fueron elaboradas sobre la base de un artículo publicado por Fenwick et al. (2011) cuyas preguntas fueron modificadas, adaptadas y traducidas para este estudio. La encuesta fue validada por un grupo de expertos, por lo que al ser un instrumento elaborado y validado, el mismo queda a disposición para ser aplicado en otras poblaciones de estudiantes y usarse como referencia para futuros trabajos de investigación.

Se encontró un adecuado nivel de respuestas correctas en relación a la función del investigador y los alcances del comité de ética, así como a las Normas CIOMS; lo cual refleja el interés que presentan los estudiantes por los aspectos relacionados a la investigación. Sin embargo, esta información debe reforzarse constantemente debido a que las instituciones, normas y reglamentos se encuentran en continua evolución.

Se encontró un alto nivel de desconocimiento en las preguntas referidas a las Tres Rs, el punto final y el organismo regulador de la investigación con animales; esto probablemente se deba a que estos temas puntuales no son impartidos con el énfasis necesario en el plan de estudios dando prioridad a otras áreas de la profesión. Esta realidad es similar a lo encontrado en otros países tal como lo mencionan Fenwick et al. (2011), quienes para superar este déficit, decidieron implementar cursos y módulos de más de una hora sobre ética en escuelas veterinarias.

Los resultados muestran que los estudiantes de medicina veterinaria tienen actitudes positivas frente a la ética en investigación, lo cual debería ser aprovechado por los educadores, implementando foros, simposios y jornadas que permitan generar momentos de discusión y análisis para fortalecer estas actitudes no dejando de lado la transmisión de conocimientos que refuerce el proceder ético de los estudiantes; ello se traducirá en beneficios para el bienestar de los animales como de la calidad científica, formando mejores investigadores.

En cuanto a dejar de tomar decisiones sobre el bienestar animal bajo condiciones económicas, los estudiantes de todos los años respondieron positivamente, especialmente los estudiantes de primeros años más que los de cuarto y quinto. En el Reino Unido, Levine, Mills y Houpt (2005) obtuvieron resultados negativos en una pregunta similar, lo que desencadeno que la Asociación Americana de 
Medicina Veterinaria (AVMA) promocionará políticas que orientaron a que los Médicos Veterinarios reafirmen el bienestar de los animales desde un punto de vista moral, ético y filosófico.

Con respecto a la pregunta sobre utilizar un número de truchas arcoíris mayor a la que propone el estadístico, se obtiene resultados similares que lo antes discutido, ya que los estudiantes del primer año están en desacuerdo en un $88.3 \%$ mientras que los de quinto año sólo en un $45,8 \%$. Esto indica nuevamente que los estudiantes del primer año son más sensibles que los estudiantes del último año (Levine et al., 2005). Estos resultados podrían deberse a que durante la formación se utilizan animales de estudio para los cursos de anatomía, farmacología, semiotecnia, fisiología, etc. lo cual origina que cuando llegan al quinto año "se les hace común" trabajar en clases prácticas con animales y se ven presionados a continuar debido a los requerimientos y exigencias del plan de estudios.

Sin embargo, en la siguiente pregunta en donde se cambia la especie animal, los estudiantes de quinto año que contestaron usar un número mayor de truchas arcoíris mantienen su posición aun cuando se tratase de utilizar perros, lo que indica una actitud negativa hacia preservar la vida del animal, inclusive no se ven influenciados por la especie. Se hace necesario mejorar ciertos conocimientos que orienten a una mejor actitud del estudiante, según Cardozo et al. (2007), gracias a la definición de las condiciones genéticas y medioambientales, se está concientizando a los investigadores de usar algunas especies más que otras; en la actualidad se usan en un $85-90 \%$ ratas, ratones y otros roedores, y los perros y gatos en menos de $1 \%$.

Un punto importante y muy discutido por diferentes organizaciones, instituciones y grupos es la utilización de animales en las clases prácticas con el objetivo de que el alumno adquiera experiencia con el material fresco, el cual será usado para cirugías o necropsias. En la pregunta referida al usar material fresco sin importar el número de animales sacrificados, se observó una actitud positiva por parte de los estudiantes de todos los años; sin embargo, se observa una tendencia a disminuir conforme se avanza en los años de estudio, ya que los estudiantes de primer y tercer año están en desacuerdo en un $71,4 \%$ y $75,6 \%$, respectivamente; mientras que los de quinto año solo en un $58,3 \%$.

Estos resultados reflejan una pérdida de sensibilidad del estudiante durante su proceso de formación, ello podría ser reforzado inculcándoles la importancia de usar el número adecuado de animales, brindado las mejores condiciones de hábitat y manejo. Paul y Podberscek (2000) encontraron también que los estudiantes del último año tuvieron niveles más bajos de sensibilidad que los ingresantes respecto al bienestar hacia los perros, gatos y vacas.

No se encontró diferencia sobre los conocimientos de aspectos éticos en investigación según sexo, sin embargo si en los resultados de actitudes. Los hombres son menos sensibles que las mujeres y tienen un mayor porcentaje de no saber o no responder ante el dilema ético presentado, lo cual concuerda con un estudio realizado en el Reino Unido, en donde Levine et al., (2005) encuentran que los hombres presentan un nivel menor de empatía hacia los animales que las mujeres. A estas conclusiones también llegan autores como Heleski, Mertig y Zanella (2004), Serpell (2005) y, de Boo y Knight (2005). Cabe resaltar que la carrera de Medicina Veterinaria en la actualidad tiene una mayor proporción de estudiantes mujeres (Paredes y Zuazo, 2014).

La priorización del bienestar animal por sobre el factor económico no se ve afectado por el género del estudiante. En este punto influye mucho la base ética del investigador. Existen centros e instituciones que apoyan esta iniciativa, y se evalúa brindar fondos a quienes cumplan con los principios del bienestar animal en sus investigaciones. Bajo esta norma, los estudiantes investigadores no decidirían entre el bienestar de un ser o el factor económico que implica. Esto también ocurre en los comités de ética que regulan la aplicación de principios éticos en investigación con animales.

Se considera necesario fomentar el principio de las Tres Rs en las facultades de Medicina Veterinaria para formar estudiantes investigadores integrales (Couceiro-Vidal, 2008). Asimismo, este tipo de estudios debería servir como una iniciativa para realizar nuevos estudios sobre a la ética con animales de producción, praxis de los Médicos Veterinarios en animales menores, impulsar los cinco principios del bienestar animal (animales libres de sed y hambre; libres de incomodidad, libres de dolor, lesiones y enfermedad, libertad de expresar su comportamiento normal, libres de miedo y angustia), decisión de ejecución de proyectos necesarios e innecesarios; alternativas de uso experimental; regulaciones nacionales e internacionales, etc. 
Esta investigación aporta un diagnostico preliminar acerca del panorama de conocimientos y actitudes que presentan estudiantes de la carrera de Medicina Veterinaria respecto a la investigación con animales, a partir del cual se podrá potenciar las actitudes e incrementar los conocimientos por medio de cursos orientados a sembrar y fortalecer las bases éticas en busca de mejorar la formación de investigadores.

\section{CONCLUSIONES}

Los conocimientos acerca de las Tres Rs de Burch y Russel, punto final y acerca del comité de ética institucional requieren ser reforzados dentro de la formación universitaria de los estudiantes de Medicina Veterinaria.

Los estudiantes indicaron que la especie animal con la que se trabaja en investigación no influye en la decisión de reducir o no los tamaños de muestra en estudios de investigación.

Los estudiantes mujeres tienen una actitud positiva y son más sensibles que los hombres frente a los temas relacionados a la ética en investigación con animales.

Los estudiantes de primer año tienen mejor actitud y mayor sensibilidad a los temas de ética en investigación con animales.

\section{Correspondencia:}

Melanie Caballero García

Correo electrónico: melacg11@gmail.com

\section{REFERENCIAS BIBLIOGRAFICAS}

1. Cardozo de Martínez, C. A. \& Mirad de Osorio, A. (2015). Ética en investigación con animales: una actitud responsable y respetuosa del investigador con rigor y calidad científica. Revista Latinoamericana de Bioética, 8(15), 46-71.

2. Cardozo de Martínez, C. A., Mrad de Osorio, A., \& Martínez, C. (2007). El animal como sujeto experimental: Aspectos técnicos y éticos. Santiago de Chile: Universidad de Chile.

3. Carlsson, H. E., Hagelin, J., Höglund, A. U., \& Hau, J. (2001). Undergraduate and postgraduate students' responses to mandatory courses (FELASA category C) in laboratory animal science. Laboratory animals, 35(2), 188-193.

3. Couceiro-Vidal, A. (2008). Enseñanza de la bioética y planes de estudios basados en competencias. Educación médica, 11(2), 69-76.

4. de Boo, J., \& Knight, A. (2005). "Concepts in animal welfare": a syllabus in animal welfare science and ethics for veterinary schools. Journal of Veterinary Medical Education, 32(4), 451-453.

5. Fenwick, N., Danielson, P., \& Griffin, G. (2011). Survey of Canadian animal-based researchers' views on the Three Rs: replacement, reduction and refinement. PLoS One, 6(8), e22478.

6. Fernández, J., \& Heuze, Y. M. (2007). El programa interno para el cuidado y uso de los animales de laboratorio en las instituciones biomédicas docentes, de investigación científica e industria farmacéutica. Acta bioethica, 13(1), 17-24.

7. Heleski, C. R., Mertig, A. G., \& Zanella, A. J. (2004). Assessing attitudes toward farm animal welfare: A national survey of animal science faculty members 1. Journal of Animal Science, 82(9), 2806-2814.

8. Howard, B., Hudson, M., \& Preziosi, R. (2009). More is less: reducing animal use by raising awareness of the principles of efficient study design and analysis. Alternatives to laboratory animals: ATLA, 37(1), 33-42.

9. Huertas, S. M. (2009). El bienestar animal: un tema científico, ético, económico y político. Agrociencia, 13(3), 45-50.

10. Kimwele, C., Matheka, D., \& Ferdowsian, H. (2011). A Kenyan perspective on the use of animals in science education and scientific research in Africa and prospects for improvement. Pan African Medical Journal, 9(1), 45.

11. Kitto, S. L., Griffiths, L. G., \& Pesek, J. D. (2003). A long-term study of knowledge, risk, and ethics for students enrolled in an introductory biotechnology course 1 2. Journal of animal science, 81(5), 1348-1353.

12. Leenaars, M., Savenije, B., Nagtegaal, A., van der Vaart, L., \& Ritskes-Hoitinga, M. (2009). Assessing the search for and implementation of the three Rs: a survey among scientists. Altern Lab Anim, 37(3), 297-303.

13. Levine, E. D., Mills, D. S., \& Houpt, K. A. (2005). Attitudes of veterinary students at one US college toward factors relating to farm animal welfare. Journal of Veterinary Medical Education, 32(4), 481-490.

14. Lewis, D. (2009). Animal Welfare, Ethics and 3Rs: Training materials and resources. 1ed Leeds, England: University of Leeds.

15.Lolas, F., Quezada, A., \& Rodriguez, E. (2006). Investigación en salud dimensión ética. Santiago de Chile: Andros Impresores. 
16. Main, D. C. J., Thornton, P., \& Kerr, K. (2005). Teaching animal welfare science, ethics, and law to veterinary students in the United Kingdom. Journal of Veterinary Medical Education, 32(4), 505-508.

17. Paredes, A., \& Zuazo, J. (2014). Percepción de los estudiantes de medicina veterinaria acerca de los perros vagabundos y sus estrategias de control en Lima, Perú. Salud Tecnol. Vet. 2, 113-119.

18. Paul, E. S., \& Podberscek, A. L. (2000). Veterinary education and students' attitudes towards animal welfare. The Veterinary Record, 146(10), 269-272.

19. Perry, P. (2007). The ethics of animal research: a UK perspective. ILAR journal, 48(1), 42-46.
20.Self, D. J., Baldwin Jr, D. C., Wolinsky, F. D., \& Shadduck, J. A. (1993). Further exploration of the relationship between veterinary medical education and moral development. J Vet Med Educ, 20(3), 140-7.

21.Serpell, J. A. (2005). Factors influencing veterinary students' career choices and attitudes to animals. Journal of veterinary medical education, 32(4), 491-496.

22. Smith, J. A., \& Jennings, M. (1998). Ethics training for laboratory animal users. Laboratory animals, 32(2), 128-136. 